\title{
Painel de indicadores de resíduos para atividades industriais: Balanced Scorecard Management of Waste (BSMW)
}

Panel indicators of industrial activities for waste: balanced scorecard of waste management (BSMW)

\author{
Carlos Enrique De Medeiros Jerônimo', Luciana Fentanes² \\ ' Doutorado em Engenharia Química, Engenheiro de Processamento de Petróleo da Petrobras, Universidade Potiguar, Natal, Brasil. \\ 2 Epecialista em Gestão e Perícias Ambientais, Bióloga, UNI-RN, Natal, Brasil.
}

\section{Resumo}

Este trabalho apresenta uma proposta de um painel de indicadores, para avaliação do desempenho do gerenciamento dos resíduos sólidos industriais, denominado de Balanced Scorecard Management of Waste (BSMW). Esse elemento foi elaborado com base em 17 indicadores, que aferem dados relativos a geração e coleta dos resíduos; gestão da estrutura física de suporte; tratamento e destinação final; gestão de custos e atendimento a cenários acidentais. Os resultados foram aferidos em uma organização de grande porte do segmento petroquímico, tendo-se ao final de um ano de monitoramento dos dados um resultado para o índice global da qualidade do gerenciamento, BSMW, igual a três. O que representa uma imaturidade na gestão do referido empreendimento, e uma aderência aos elementos da política da gestão ambiental da organização. O modelo desenvolvido foi considerado aceitável e configura-se como uma fonte para estudos comparativos entre diferentes filiais ou organizações.

Palavras-chave: Resíduos sólidos, BSMW, gerenciamento e indicadores.

\begin{abstract}
This paper presents a proposal for a panel of indicators for assessing the performance of industrial solid waste management, called the Balanced Scorecard Management of Waste (BSMW). This element was based on 17 indicators that measure data on waste generation and collection, management of physical infrastructure support, treatment and disposal, cost management and treatment of accidental scenarios. The results were measured in a large organization of the petrochemical sector, and it was the end of one year of monitoring data results to a global index of quality of management, BSMW equal to three. What is an immaturity in the management of that enterprise, and an adherence to the policy elements of the organization's environmental management. The model was considered acceptable and is configured as a source for comparative studies between different branches or organizations.
\end{abstract}

Keywords: Solid waste, BSMW, management, and indicators. 


\section{INTRODUÇÃO}

As preocupações com a temática ambiental, sobretudo, associadas ao conceito de sustentabilidade vêm ecoando como um elemento recorrente nas pesquisas e textos acadêmicos que tratam do assunto. No tocante ao gerenciamento de resíduos sólidos não é diferente, visto que esse é um dos principais problemas ambientais associado a qualquer atividade industrial.

Consequentemente, a geração continuada dos resíduos sólidos nas grandes indústrias brasileiras constitui um desafio para as administrações e definição das políticas ambientais.

Segundo Soares (2004), embora a legislação ambiental exista no país, praticamente não há regras específicas sobre o estabelecimento de padrões de qualidade e disposição final de resíduos. A responsabilidade por dano ambiental e a definição de competências administrativas, não têm recebido um tratamento legal e institucional capaz de amenizar ou reverter à situação encontrada na maioria dos municípios brasileiros.

Diante da problemática crescente do acúmulo de resíduos sólidos principalmente nas atividades em indústrias petroquímicas, fica evidente a necessidade premente de medidas de controle da geração desses rejeitos. Para tanto, impulsionadas pelas exigências dos processos de certificações internacionais, as organizações necessitam desenvolver ações planejadas e integradas quanto à gestão e gerenciamento desses resíduos e implementar a legislação pertinente ao setor.

$\mathrm{Na}$ indústria petroquímica, a importância do gerenciamento eficaz dos resíduos ainda é mais potencializada, visto que a maioria dos resíduos gerados apresenta na sua composição efeitos tóxicos, alta solubilidade e reatividade, que levam a classificá-los como resíduos perigosos, conforme a NBR10004, classe I.

Logo, um difícil pilar na estruturação do modelo de gestão dessas organizações se eleva, relacionado a qualificação das informações da geração e gerenciamento dos resíduos gerados nas atividades industriais. Que apesar da imposição legal do inventário de resíduos anual, ainda se torna um elemento incipiente na estrutura de gestão dessas organizações.

Alguns estudos são demonstrados na literatura, sobretudo que vinculam a gestão de indicadores de resíduos na indústria do petróleo, tais como: Salgado (2004), Luz et al (2006), Ferreira (2010) E Munck (2011).

O caso discutido em LUZ et al (2006) apresenta um método de medição de desempenho ambiental, que calcula um índice global entre 0 e $100 \%$, específico para uma operação, mas que pode ser comparado com outras operações, dentro e fora da indústria e, cumulativamente, em cadeias produtivas.

O estudo do caso de LUZ et al (2006), abriu frentes de interesse para pesquisa de índices ou indicadores que meçam tal qualidade da gestão. Por exemplo, o acompanhamento do índice de desempenho ambiental na mesma operação e a verificação dos impactos de ações tomadas no resultado final; a replicação do método nas demais operações num mesmo site, comparando os impactos causados pelas empresas; e a definição de importâncias relativas para cada indicador e a elaboração de um manual de aplicação deles, que pode se tornar um modelo de referência de gestão exclusivo da manufatura, independente do SGA da empresa.

Souza; Sarmento (2006) reportam que grandes empresas do segmento petroquímico atuam lado a lado cooperando e competindo, adequando-se de forma intensiva às exigências de um novo mercado cada vez mais consciente das questões ambientais. É neste cenário de competitividade e desafios que o Sistema de Gestão Ambiental, regido pelas normas da série ISO 14.000, destaca-se como parte integrante de um planejamento estratégico, com a finalidade de equilibrar a proteção ambiental e prevenção da poluição com as necessidades socioeconômicas. Diante desta realidade, esses autores disponibilizam instrumentos para avaliar do desempenho ambiental das atividades "upstream" da indústria petrolífera, tornando-se um diferencial necessário que agrega valor, auxiliando na tomada de decisões ambientais, políticas e administrativas, mantendo a competitividade da empresa e fomentando a disputa de um melhor desempenho ambiental entre as companhias. Sendo assim, tem-se uma forte carência de elementos de medição para a gestão ambiental nessas organizações.

Fazendo uma correlação da medição da eficiência da gestão em sistemas públicos, em Bringhenti et al (2006) tem-se a definição que a aplicação do Grupo de Indicadores de Referência na avaliação do Programa de Coleta Seletiva implantado no município de Vitória mostrou-se adequada e suficiente, possibilitando a identificação de informações importantes que embasaram a avaliação do andamento e dos resultados do referido Programa, sendo um método de suporte a gestão de resíduos sólidos. Sendo 
de fato, o painel de indicadores, uma boa técnica de medir a excelência do sistema de gestão.

Munck et al (2011) também demonstra a importância dos indicadores para as organizações, definindo a utilização do painel de indicadores como uma maneira de organizar uma vasta quantidade de informações sobre uma ampla variedade de riscos e oportunidades. E consideram os indicadores importantes porque eles resumem uma grande quantidade de informações de diferentes fontes em um formato fácil para entender, comparar e manipular. Pensando nas organizações, os indicadores podem contribuir por ajudar o tomador de decisão a visualizar quais ações precisam ser enfatizadas no futuro. Bem como, que para mensuração da ecoeficiência, os indicadores fornecem subsídios para criação de planos adaptados ao contexto de cada organização.

Logo, a união da estrutura organizacional na sua fronte de painéis de resultados, conforme cita Ribeiro (2011) "os projetos que tenham como objetivo utilizar resíduos como matérias-primas deve ocorrer de forma meticulosa, sistemática e de forma a reduzir ao mínimo as chances de contaminação do meio ambiente e dos seres humanos, sendo assim, os indicadores de desempenho um elemento para auxiliar na mensuração desses".

Diante desse quadro, surge um grande desafio que é o correto gerenciamento e a compilação entre o banco de indicadores (nesse difícil cenário que é a indústria petroquímica) e os elementos que produzem uma gestão eficaz é o conhecimento de dados operacionais, cenários de controle e principalmente os montantes envolvidos. Amaral (2003) já levanta tal preocupação. Configura-se, então, o primeiro desafio que é a estruturação de um painel de gestão eficaz para mensurar os diferentes níveis de geração, direcionamento para as fontes e as causas básicas para dar foco ao cenário de suporte operacional.

Sendo assim, neste trabalho é apresentada uma proposta para um painel de indicadores que suportam o gerenciamento dos resíduos sólidos em indústrias petroquímicas. Sendo considerados os efeitos das causas, técnicas de tratamento, fontes de geração, atuação estratégica dos cenários de inserção e os custos associados a tais operações.

Inspirados no conceito de BSC (Balanced Scorecard) considerada como uma nova abordagem para administração estratégica, desenvolvida por Robert Kaplan e David Norton em meados de 1990; que mede os resultados das diferentes perspectivas das organizações, o referido painel de resíduos será denominado de BSMW, ou Balanced Scorecard Management of Waste. Permitindo com isso, efeitos comparativos entre organizações, suas filiais ou segmentos diferentes para uma mesma base de referência.

\section{MÉTODO}

O desenvolvimento do painel foi realizado mediante a imersão para acompanhamento das rotinas operacionais de uma indústria de processamento de derivados petroquímicos para fins como combustíveis, durante seis meses de atividades normais.

Foram realizados levantamentos das estruturas de suporte utilizadas e dos elementos ligados ao gerenciamento dos resíduos da organização.

As principais características do painel, seguem no quadro abaixo:

\begin{tabular}{|c|l|}
\hline Porte & $\begin{array}{l}\text { Empresa de grande porte, com mais de } 1000 \text { colaboradores e faturamento que } \\
\text { a classifica contabilmente como grande empresa. }\end{array}$ \\
\hline Área & $\begin{array}{l}\text { A área total da organização é superior a } 900.000 \mathrm{~m}^{2} \text {, tendo-se cerca de } \\
100.000 \mathrm{~m}^{2} \text { de área construída. }\end{array}$ \\
\hline Resíduos Gerados & $\begin{array}{l}\text { Industriais (classe I) e resíduos associados as atividades administrativas e de } \\
\text { áreas comuns. }\end{array}$ \\
\hline Forma da Coleta & $\begin{array}{l}\text { Recolhimento em pontos específicos, dotados de contenção e recipientes } \\
\text { segregados por tipo de resíduo. O processo é feito por equipe especializada, } \\
\text { por meio de contrato específico para tal fim. }\end{array}$ \\
\hline Tratamento & $\begin{array}{l}\text { A empresa possui estrutura própria de tratamento de resíduos oleosos por } \\
\text { meio de um landfarming controlado. Os demais resíduos são destinados para } \\
\text { empresas de coprocessamento, incineração, reciclagem e aterro municipal. }\end{array}$ \\
\hline Armazenamento temporário & $\begin{array}{l}\text { Dispõe de um galpão para triagem, acondicionamento, trituração, } \\
\text { enfardamento dos principais resíduos gerados, sobretudo, recicláveis. }\end{array}$ \\
\hline Disposição Final & $\begin{array}{l}\text { A destinação final dos resíduos é toda feita por meio da contratação de } \\
\text { serviços de transporte e da prestação de serviços especializado. }\end{array}$
\end{tabular}




\begin{tabular}{|c|c|}
\hline $\begin{array}{c}\text { Serviços relacionados a } \\
\text { biodiversidade }\end{array}$ & $\begin{array}{l}\text { Cabe a estrutura de suporte do gerenciamento dos resíduos, a manutenção de } \\
\text { elementos que estão vinculados a conservação da biodiversidade, tais como: } \\
\text { podação, recolhimento de animais silvestres em áreas inapropriadas, soltura } \\
\text { de animais em áreas verdes, capina, conservação de áreas e monitoramento } \\
\text { ambiental. }\end{array}$ \\
\hline Estrutura disponível & $\begin{array}{l}\text { Para desenvolvimento dos serviços a organização dispõe de galpões (que } \\
\left.\text { totalizam área superior a } 10000 \mathrm{~m}^{2}\right), 2 \text { viaturas de carga, equipamentos } \\
\text { (prensa, trituração e balanças), equipamentos para varrição de ruas, equipe de } \\
\text { auxiliares de serviços gerais e cerca de } 60 \text { colaboradores são envolvidos nestas } \\
\text { atividades. }\end{array}$ \\
\hline Cenário de Inserção & $\begin{array}{l}\text { A indústria encontra-se inserida a margem de um grande rio, e é cortada por } \\
\text { fragmentos florestais urbanos e por pequenos riachos ao longo de toda sua } \\
\text { extensão. É servida por uma rica biodiversidade, incluindo espécies } \\
\text { ameaçadas de extinção. }\end{array}$ \\
\hline Certificações & ISO 9001, 14001 e OSHAS 18001. \\
\hline
\end{tabular}

Para definição do painel de indicadores foram considerados os atributos e requisitos, conforme recomendado por RAMOS (2009), a saber:

\begin{tabular}{|c|l|}
\hline Atributos & $\begin{array}{l}\text { Simplicidade, validade, estabilidade, sensibilidade, cobertura, independência, } \\
\text { confiabilidade, custo, obtenção e periodicidade. }\end{array}$ \\
\hline Requisitos & $\begin{array}{l}\text { Fácil compreensão, estável, alta sensibilidade, abrangência regional ou local, } \\
\text { independente, confiável, baixo ou médio custo, existe fontes para coleta dos } \\
\text { dados e avaliação anual. }\end{array}$ \\
\hline Restrições & $\begin{array}{l}\text { Dados secundários e por correlações com dados da literatura. Todos os } \\
\text { indicadores devem ser obtidos por meio de dados diretos da organização. }\end{array}$ \\
\hline
\end{tabular}

Com base nisso, foi construído o painel. E as medições foram alinhadas a política ambiental da organização e seus objetivos/metas, frente ao sistema de gestão integrada.

\section{APRESENTAÇÃO E ANÁLISE DOS DADOS}

Considerando os elementos principais do processo produtivo da organização, utilizada para o desenvolvimento do painel de indicadores, foi possível estruturar os elementos principais da gestão de resíduos na organização, traçando-se uma subdivisão conforme as atribuições. Logo, segue no fluxograma abaixo, tal modelo desenvolvido, elencando as principais referências aos objetivos e metas do sistema de gestão ambiental da organização:

Nestas subdivisões, o entendimento das premissas associadas a gestão unitária de cada fluxo

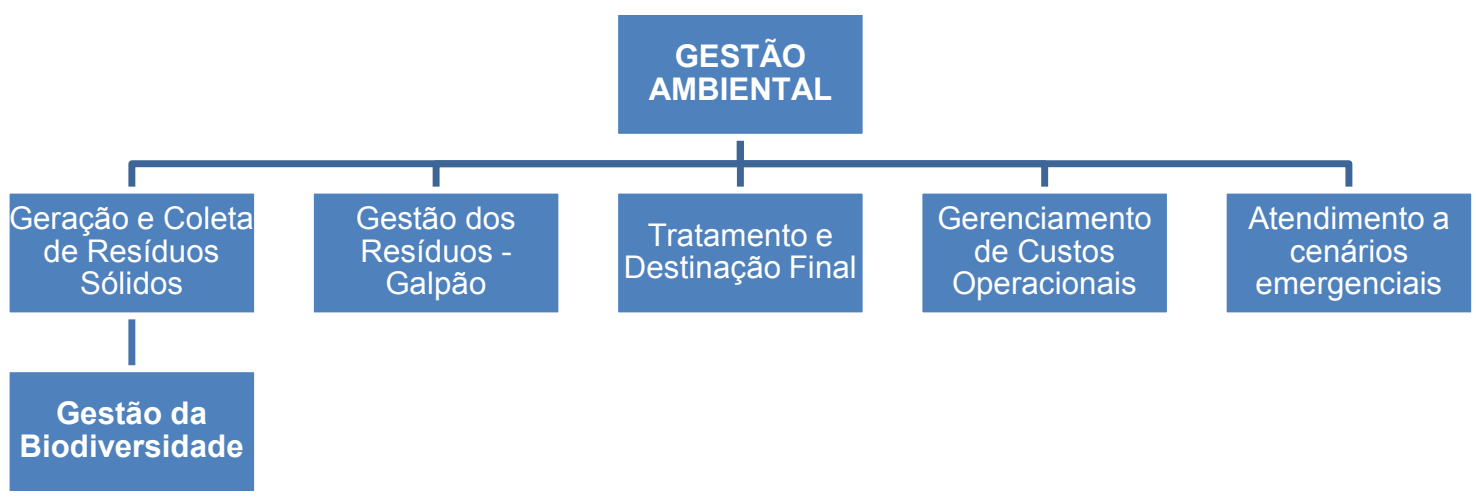


do processo de gerenciamento ambiental de uma organização, enquanto modelagem dos dados operacionais é descrito como:

Geração e Coleta dos Resíduos: conhecimento dos locais da geração dos resíduos, qualidade e quantidade dos resíduos gerados, acondicionamento nos pontos de geração, definição da eficácia das rotinas de coleta, formas e métodos de coleta, dimensionamento da estrutura de coleta e qualidade percebida pelos usuários desses serviços. Inclui-se nestas atribuições a gestão da estrutura de biodiversidade da organização;

Gestão da estrutura física de suporte: gestão de estoques, estrutura de contenção, ordem e limpeza, dimensionamento de recursos materiais, gestão de insumos (água e energia elétrica) e aproveitamento das áreas disponíveis;

Tratamento e Destinação Final: cumprimento da legislação e normas associadas, seguridade ambiental, eficiência das técnicas de tratamento, manutenção do acervo documental (legal) e verificação de fornecedores;

Gestão de custos: apropriação de custos operacionais, custos da infra-estrutura de suporte, custos fixos, despesas administrativas e vinculo com os níveis de eficiência da organização;

Atendimento a cenários acidentais: prevenção de acidentes, custos associados a sinistros, matriz de responsabilidades das causas associados a acidentes ambientais, suporte de dados a prêmios de seguradoras e dimensionamento das estruturas de contingência.

Diante das premissas e objetivos da organização, a mensuração dos resultados foi composta por meio do seguinte elenco de indicadores:

Tabela 1: Metas e Cenário Ideal para cada um dos Indicadores.

\begin{tabular}{|c|l|}
\hline $\begin{array}{c}\text { Geração e Coleta dos } \\
\text { Resíduos }\end{array}$ & $\begin{array}{l}\text { Indicador do Grau de Periculosidade dos Resíduos (IGPR), } \\
\text { Indicador da Qualidade da Coleta Seletiva (IQCS), Reciclagem } \\
\text { de Resíduos Classe I (RRC1), Gestão de Resíduos Perigosos } \\
\text { (GRP), Resíduos Construção Civil (RCC), Produção de } \\
\text { Resíduos (PR) e Número de Impacto a Fauna (NIF). }\end{array}$ \\
\hline $\begin{array}{c}\text { Gestão da estrutura } \\
\text { física de suporte }\end{array}$ & $\begin{array}{l}\text { Indicador de Ordem e Limpeza do Galpão de Resíduos (IOLG) } \\
\text { e Custo de Manutenção do Galpão (CMG). }\end{array}$ \\
\hline $\begin{array}{c}\text { Tratamento e } \\
\text { Destinação Final }\end{array}$ & $\begin{array}{l}\text { Indicador da Gestão do Landfarming (IGL), Produtividade do } \\
\text { Landfarming (PL), Custeio do Landfarming (CL), Indicador de } \\
\text { Qualidade dos Fornecedores (IQF) e Custo do Tratamento de } \\
\text { Resíduos (CTR). }\end{array}$ \\
\hline Gestão de custos & $\begin{array}{l}\text { Indicador Global de Custos (IGC), Indicador de Custos } \\
\text { Operacionais (ICO) e Impacto da Coleta Seletiva nos Custos } \\
\text { (ICSC). }\end{array}$ \\
\hline $\begin{array}{c}\text { Atendimento a } \\
\text { cenários acidentais }\end{array}$ & $\begin{array}{l}\text { Produtividade da Estrutura de Contingência (PEC) e o Índice } \\
\text { de Acionamento em Sinistros (IAS) }\end{array}$ \\
\hline
\end{tabular}

As identidades dos indicadores e mecanismos de medição são descritas no quadro a seguir, considerando o método de calculo e a tendência para o melhor cenário, tendo indicando que quanto maior o valor do indicador, melhor, e $\mathbf{D}$ indicando que quanto menor o indicador, melhor.

Tabela 2: Metas e Cenário Ideal para cada um dos Indicadores.

\begin{tabular}{|c|l|c|}
\hline INDICADOR & \multicolumn{1}{|c|}{ DESCRIÇÃO } & TENDENCIA \\
\hline & $\begin{array}{l}\text { Consiste na relação do custo total da operação do } \\
\text { Landfarming e a carga de resíduos retirada no mês. } \\
\text { Esse indicador deve ser utilizado para cálculos }\end{array}$
\end{tabular}




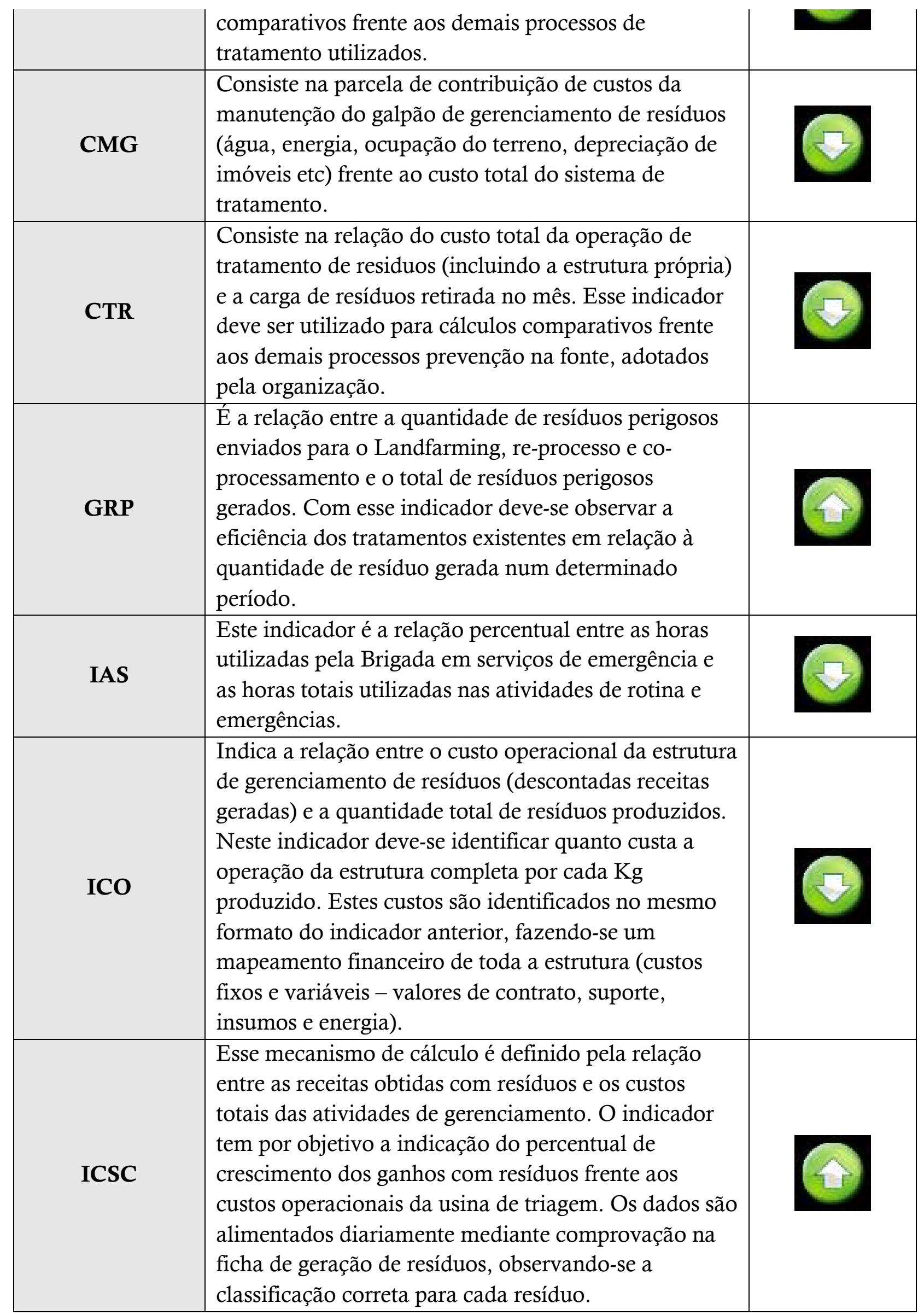




\begin{tabular}{|c|c|}
\hline IGL & $\begin{array}{l}\text { Esse indicador consiste na determinação da qualidade } \\
\text { da gestão operacional do landfarming, considerando a } \\
\text { média dos percentuais de irrigações realizadas ante as } \\
\text { planejada, a umidade obtida (essas medidas são feitas } \\
\text { com base na coleta no período da manhã, e em análise } \\
\text { realizadas em balança de infravermelho devidamente } \\
\text { calibradas) antes da irrigação e os valores ideais para } \\
\text { uma nova irrigação, sendo um parâmetro de controle } \\
\text { dos níveis de eficiência do sistema. e o percentual de } \\
\text { aerações realizadas frente as planejadas. Sendo assim, } \\
\text { de forma a manter o sistema em um regime de controle } \\
\text { o cumprimento dessa rotina é imprescindível. }\end{array}$ \\
\hline IGPR & $\begin{array}{l}\text { Consiste na relação entre a quantidade de resíduos } \\
\text { perigosos (classe I) e o total de resíduos gerados na } \\
\text { organização. Com esse indicador deve-se identificar a } \\
\text { freqüência e quantidade da geração de resíduos } \\
\text { perigosos no mês. Alimenta-se diariamente a planilha } \\
\text { retirando o resultado das fichas de geração dos resíduos } \\
\text { classificados como perigosos, nos quais ocorreu } \\
\text { intervenção ou foram direcionados para a unidade de } \\
\text { triagem, no período, bem como, classifica-se a técnica } \\
\text { de tratamento adotada para os mesmos. }\end{array}$ \\
\hline IOLG & $\begin{array}{l}\text { Consiste em demonstrar o percentual de conformidades } \\
\text { listadas na aplicação da Lista de Verificação do galpão } \\
\text { (checklist) a cada } 2 \text { dias. Para isso, deve ser aplicado } \\
\text { um questionário padrão com as principais orientações a } \\
\text { serem seguidas na organização do galpão. Destes } \\
\text { calcula-se o percentual de conformidade, } \\
\text { desconsiderando no total de itens as questões não- } \\
\text { aplicáveis. Este indicador tem como objetivo verificar o } \\
\text { cumprimento das conformidades, bem como os } \\
\text { responsáveis e prazos relacionados às não- } \\
\text { conformidades em tempo definido. Devendo-se manter } \\
\text { os valores ótimos maximizados, em termos da } \\
\text { conformidade. }\end{array}$ \\
\hline IQCS & $\begin{array}{l}\text { Demonstra o percentual de resíduos que efetivamente } \\
\text { são encaminhados para reciclagem frente a quantidade } \\
\text { de resíduos recicláveis recebidos (contaminados ou não } \\
\text { contaminados). Os dados são compilados a partir das } \\
\text { fichas de geração de resíduos e a pesagem líquida no ato } \\
\text { do recebimento e após a segregação para recicláveis. O } \\
\text { peso do resíduo é líquido, onde tara-se a balança com o } \\
\text { peso do recipiente ou ocorrendo grandes quantidades } \\
\text { com o caminhão vazio. Este indicador tem como } \\
\text { objetivo a verificação da eficiência do tratamento para } \\
\text { recicláveis, em especial no processo de segregação na } \\
\text { fonte. }\end{array}$ \\
\hline
\end{tabular}




\begin{tabular}{|c|c|}
\hline NIF & $\begin{array}{l}\text { Este indicador identifica a quantidade de animais } \\
\text { mortos, contabilizados pela estrutura de recolhimento } \\
\text { nas ocorrências de chamados. Esses dados coletados } \\
\text { são registrados pelas fichas de ocorrências da Brigada e } \\
\text { alimentados diretamente na Planilha correspondente, } \\
\text { dentro do Painel de Resíduos. }\end{array}$ \\
\hline PL & $\begin{array}{l}\text { Consiste na relação entre a quantidade de resíduos } \\
\text { retirados e o total adicionado para tratamento no } \\
\text { sistema landfarming. Esse indicador deve ser } \\
\text { considerado para o dimensionamento do } \\
\text { encaminhamento de materiais para o sistema, frente a } \\
\text { capacidade máxima acumulada do equipamento. De } \\
\text { forma a evitar sobrecargas e haver um desdobramento } \\
\text { frente ao controle das variáveis dos pontos de geração. } \\
\text { Em especial, em procedimentos como: limpezas de } \\
\text { tanques, canaletas etc. }\end{array}$ \\
\hline PR & $\begin{array}{l}\text { Consiste no somatório da massa de resíduos gerados } \\
\text { por mês, registrado na base de dados que subsidia o } \\
\text { inventário anual de resíduos. }\end{array}$ \\
\hline RCC & $\begin{array}{l}\text { Este indicador demonstra o percentual da relação entre } \\
\text { a quantidade de resíduos gerados e a quantidade de } \\
\text { resíduos estimada, evidenciada em cálculo prévio na } \\
\text { ocasião do projeto. Estes valores devem constar na } \\
\text { análise de prevenção de risco gerada para a obra. }\end{array}$ \\
\hline RRC1 & $\begin{array}{l}\text { Consiste na relação entre o tratamento via re-processo e } \\
\text { coprocessamento (reciclagem) e o total de resíduos } \\
\text { gerados. Com esse indicador identifica-se o percentual } \\
\text { de resíduo perigoso que foi tratado sobre a quantidade } \\
\text { total gerada. Os dados são alimentados diariamente, } \\
\text { identificando a classificação correta e a quantidade } \\
\text { expedida para tratamento, mediante o documento } \\
\text { gerado pelo inventário de resíduos, por meio dos } \\
\text { manifestos de Resíduos. }\end{array}$ \\
\hline IQF & $\begin{array}{l}\text { Consiste no resultado médio das notas geradas a partir } \\
\text { de um checklist padrão, para avaliação dos } \\
\text { fornecedores, quanto ao cumprimento de requisitos } \\
\text { legais e técnicos, no tratamento e destinação final dos } \\
\text { resíduos. }\end{array}$ \\
\hline
\end{tabular}

Alguns fatores contribuem na estrutura física para que os indicadores sejam monitorados, e entre elas o modelo utilizado pela organização utilizada como referência para definição do banco de indicadores.

Nesse caso, esta indústria dispõe de pontos de coleta distribuídos ao longo de toda a área de produção, onde o recolhimento é feito diariamente por uma equipe denominada de "Estrutura de recolhimento". A estrutura de acondicionamento da organização é localizada externamente a área industrial, tendo-se obrigatoriamente para liberação de resíduos na portaria a geração da ficha de movimentação de resíduos no galpão (para isso, cada setor deve dispor de cerca de 4 usuários treinados no sistema). O material segue para o galpão, onde é feita a aferição da massa e as fichas da 
origem são alimentadas em banco de dados em plataforma Access, para identificação dos geradores. O material após uma nova triagem é acondicionado, e posteriormente com a geração do manifesto é encaminhado para o destino final.

Essas atividades que envolvem o tratamento de resíduos são realizadas no galpão, tendo início com o pré-armazenamento de todos os resíduos gerados, resultantes de processo ou de atividades de manutenção ou rotina, bem como o tratamento de solo contaminado direcionado para o "Landfarming". Logo, faz-se necessário conhecer técnica e economicamente todo o processo do ciclo do resíduo, desde sua geração até o tratamento final.

Como todas estas atividades demandam custos operacionais, a análise quantitativa e qualitativa de todo resíduo gerado e seu posterior tratamento e destinação requer a organização e atualização de parâmetros que viabilizem uma gestão mais focada na redução da geração de resíduos, redução de custos e otimização do processo e dos próprios contratos de serviços que lhe dão suporte.

As fontes dos dados a serem utilizados, para produção das informações que compõe o painel de indicadores são:

Inventário de resíduos, por meio da ferramenta em Access, que documenta todo resíduo gerado (bem como, os procedimentos de catalogação e coleta de informações no decorrer das atividades de gerenciamento de resíduos);

Planilha de consolidação das fichas de movimentação, para triagem dos pontos de geração dos resíduos:

Planilha de consolidação de informações para geração dos indicadores de desempenho, ora denominada de BSMW.

As principais informações a serem coletadas para registro nas planilhas de acompanhamento e geração dos indicadores são:

Controle diário dos resíduos gerados e destinados para tratamento, e suas especificações (para desdobramentos em termos de resíduos recicláveis, perigosos etc);

Lançamento de resíduos no Landfarming: freqüência semanal, agrupados a cada 5 dias e consolidados até o $25^{\circ}$ dia ou próximo dia útil;

Acompanhamento dos índices de umidade e parâmetros analíticos de controle no Landfarming - conforme coleta de amostras do solo e pré-análise realizada na própria organização: a cada 2 dias, anterior às atividades de irrigação;

Medição de variáveis para o controle de custos, tais como: consumo mensal de energia elétrica, consumo de materiais de consumo (tambores, big-bags etc), combustível de veículos, custos de tratamento de resíduos, estrutura fixa do sistema (mão-de-obra) etc.

Com base no monitoramento durante doze meses, foi possível identificar metas individuais para cada um dos indicadores, e consequentemente estabelecer o painel para definição numérica da nota de avaliação, a ser considera como a principal resposta do BSMW. Na Tabela 3 são apresentadas as informações básicas que compõe as equações individuais, com os pesos atribuídos para obtenção da nota final da gestão das empresas. Bem como, as metas de referência e o cenário ideal para cada um dos indicadores desenvolvidos.

Tabela 3: Metas e Cenário Ideal para cada um dos Indicadores.

\begin{tabular}{|c|c|c|c|c|c|c|c|c|}
\hline $\mathbf{i}$ & INDICADOR & $\mathbf{U N I D .}$ & $\mathbf{M}_{\mathbf{i}}$ & $\mathbf{B M}_{\mathbf{i}}$ & $\mathbf{C I}_{\mathbf{i}}$ & $\mathbf{M A}_{\mathbf{i}}$ & $\boldsymbol{f}_{\boldsymbol{i}}$ & $\boldsymbol{N}_{\boldsymbol{i}}$ \\
\hline $\mathbf{1}$ & $\mathbf{C L}$ & $\mathrm{R} \$ /$ ton & 3,00 & 1,60 & 0 & 0,30 & 0,05 & $\mathrm{~A}$ \\
\hline $\mathbf{2}$ & $\mathbf{C M G}$ & $\%$ & 30,00 & 10,00 & 0 & 5,00 & 0,05 & $\mathrm{~A}$ \\
\hline $\mathbf{3}$ & $\mathbf{C T R}$ & $\mathrm{R} \$ /$ ton & 4,00 & 1,00 & 0 & 1,00 & 0,20 & $\mathrm{~A}$ \\
\hline $\mathbf{4}$ & $\mathbf{G R P}$ & $\%$ & 30,00 & 90,00 & 100,00 & 5,00 & 0,05 & $\mathrm{~B}$ \\
\hline $\mathbf{5}$ & IAS & $\%$ & 5,00 & 0 & 0 & 1,00 & 0,05 & $\mathrm{C}$ \\
\hline $\mathbf{6}$ & ICO & $\mathrm{R} \$ /$ ton & 7,00 & 3,00 & 0 & 1,00 & 0,05 & $\mathrm{~A}$ \\
\hline $\mathbf{7}$ & ICSC & $\%$ & 10,00 & 3,00 & 110,00 & 2,00 & 0,05 & $\mathrm{~A}$ \\
\hline $\mathbf{8}$ & IGL & $\%$ & 95,00 & 100,00 & 100,00 & 2,00 & 0,05 & $\mathrm{~B}$ \\
\hline $\mathbf{9}$ & IGPR & $\%$ & 30,00 & 5,00 & 0 & 10,00 & 0,05 & $\mathrm{~A}$ \\
\hline
\end{tabular}




\begin{tabular}{|c|c|c|c|c|c|c|c|c|}
\hline $\mathbf{1 0}$ & IOLG & $\%$ & 99,00 & 100,00 & 100,00 & 0,50 & 0,15 & $\mathrm{~B}$ \\
\hline $\mathbf{1 1}$ & IQCS & $\%$ & 90,00 & 100,00 & 100,00 & 2,00 & 0,02 & $\mathrm{~B}$ \\
\hline $\mathbf{1 2}$ & NIF & $\begin{array}{c}\text { Animais/a } \\
\text { no }\end{array}$ & 5 & 1 & 0 & 1 & 0,02 & $\mathrm{~A}$ \\
\hline $\mathbf{1 3}$ & $\mathbf{P L}$ & $\%$ & 95,00 & 100,00 & 100,00 & 2,00 & 0,01 & $\mathrm{~B}$ \\
\hline $\mathbf{1 4}$ & $\mathbf{P R}$ & ton/mês & 100,00 & 50,00 & 0 & 20,00 & 0,05 & $\mathrm{~A}$ \\
\hline $\mathbf{1 5}$ & RCC & $\%$ & 90,00 & 100,00 & 100,00 & 5,00 & 0,05 & $\mathrm{~B}$ \\
\hline $\mathbf{1 6}$ & RRC1 & $\%$ & 20,00 & 0,00 & 0 & 10,00 & 0,05 & $\mathrm{C}$ \\
\hline $\mathbf{1 7}$ & IQF & $\%$ & 90,00 & 100,00 & 100,00 & 5,00 & 0,05 & $\mathrm{~B}$ \\
\hline
\end{tabular}

Meta da organização (M), benchmarking (BM), cenario ideal (CI), resultado do indicador (R) e margem aceitável (MA).

O BSMW é dado pela equação (1), Onde: fi é o peso do indicador, obtido através da Tabela 3. E Ni é a nota da comparação do resultado do indicador, com sua meta, conforme critério do indicador apresentado na Figura 1 (A, B ou C), e os resultados obtidos pela organização (R). E por fim, o fator que mede a qualidade da $\gamma_{i}=f\left(M_{i}, B M_{i}, C I_{i}, M A_{i}\right)$, conforme Figura 2.

$$
B S M W=\sum_{i=0}^{N} f_{i} \cdot N_{i} \cdot \gamma_{i}
$$
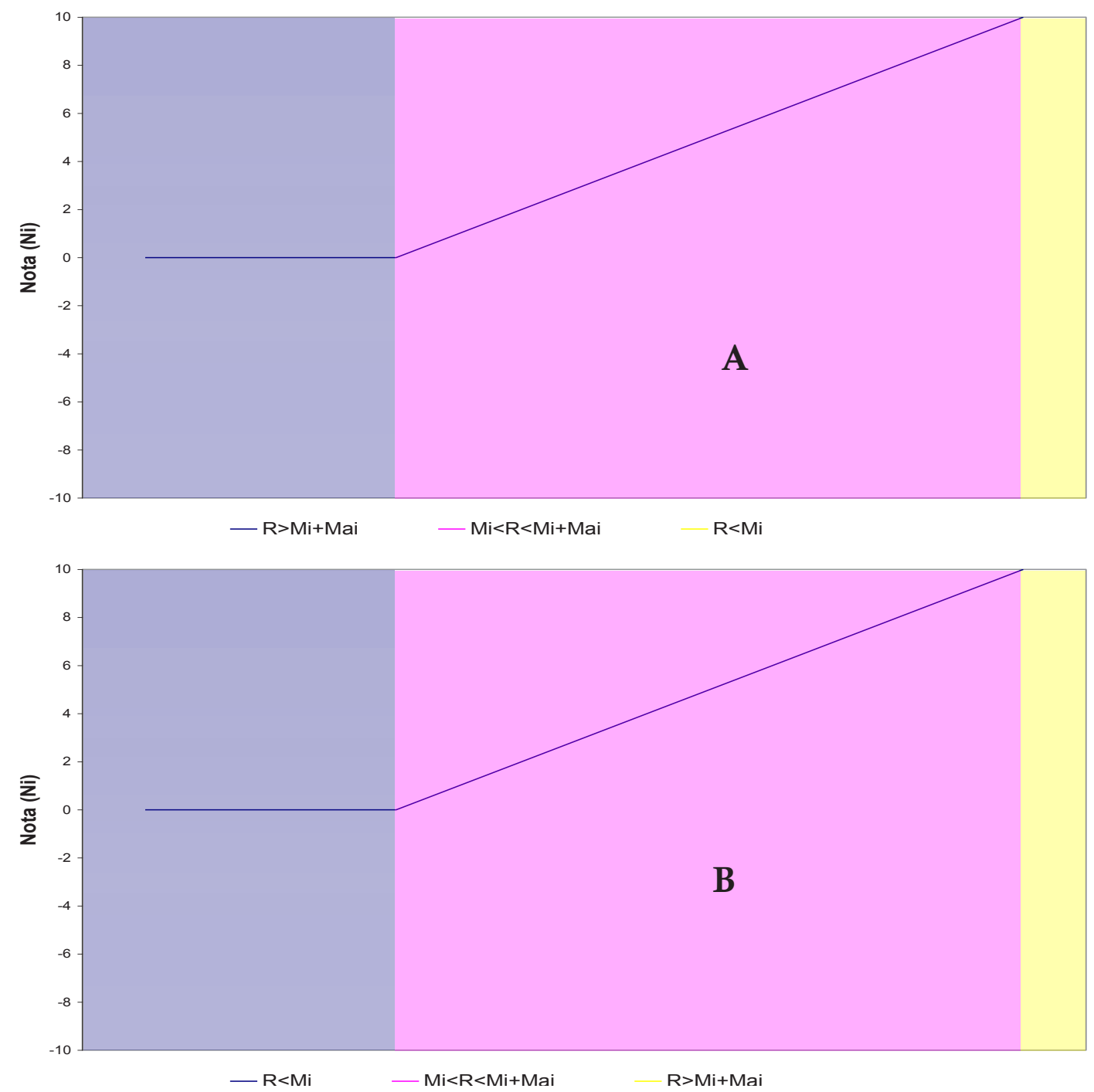


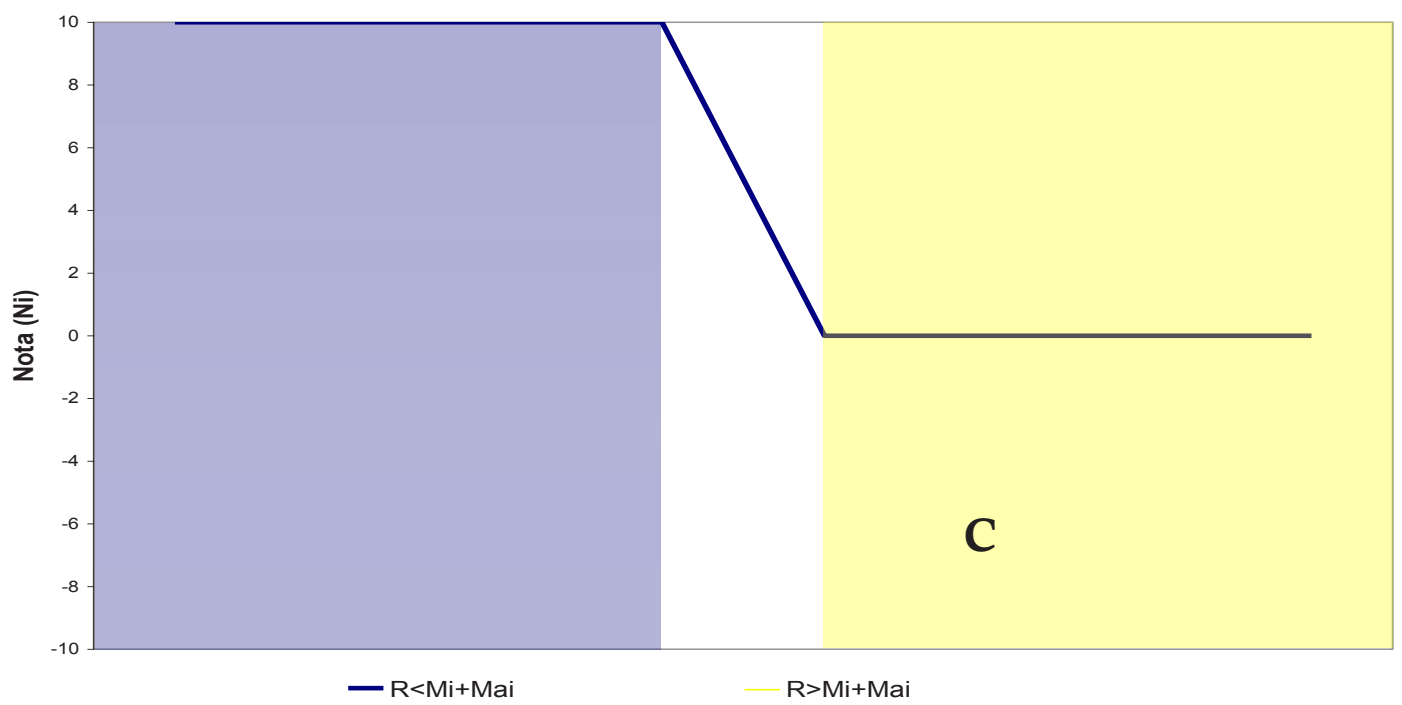

Figura 1: Método de Cálculo das Notas (Ni), com base na classificação do indicador (A, B ou C).

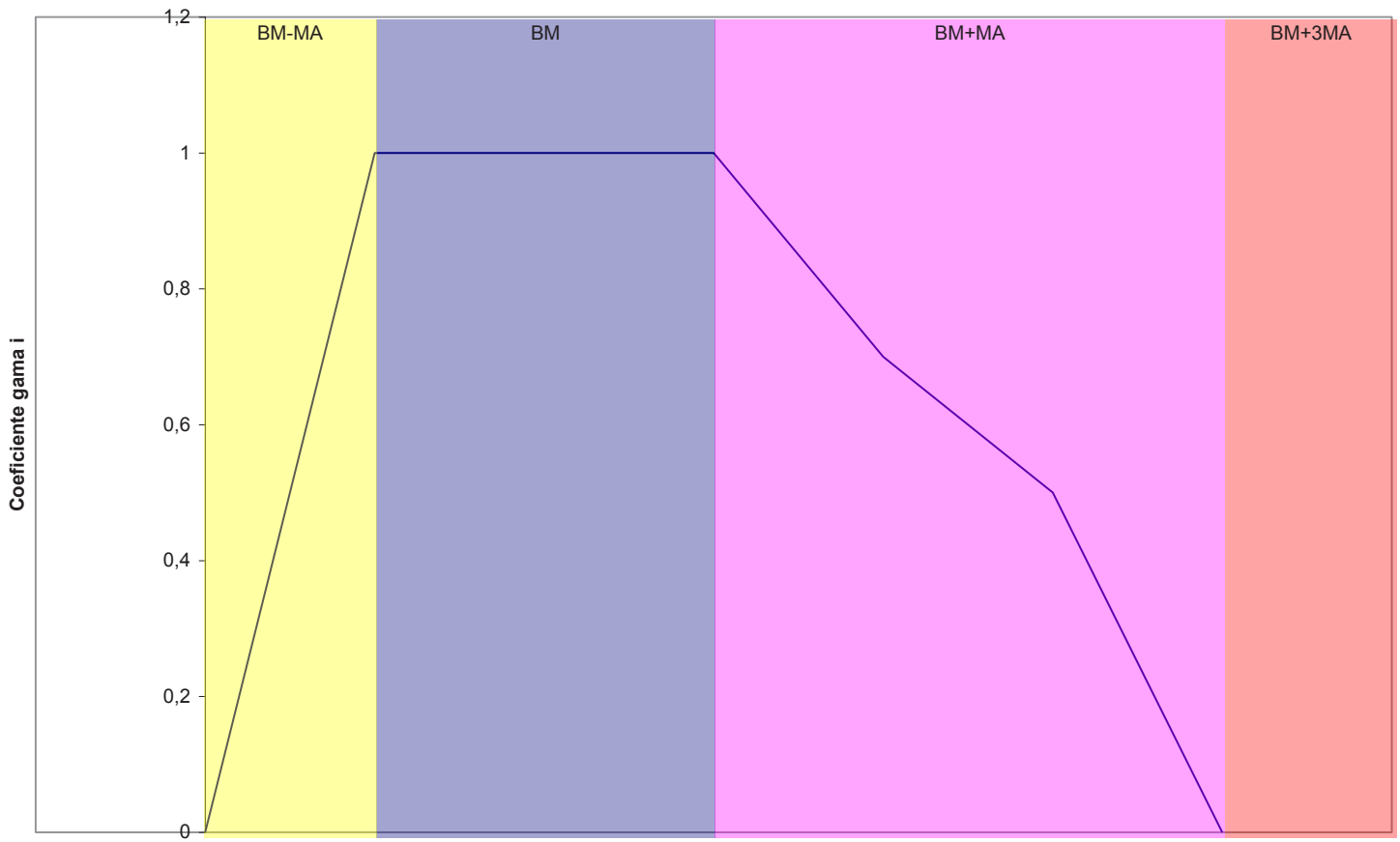

Figura 2: Determinação do coeficiente comparativo da meta as referências de Benchmarking.

Considerando o caso da indústria avaliada, considerando os resultados obtidos ao longo de um ano de monitoramento, o calculo do BSMW remeteu a valores de 3,0. Sendo considerado um valor baixo, em função da imaturidade das metas e dos resultados obtidos.

Entretanto, a metodologia apresentou-se de uma forma objetiva um elemento importante para qualificar organizações, em especial, propor melhorias aos sistemas em questão.

\section{CONSIDERAÇÕES FINAIS}

O modelo do BSMW foi desenvolvido, considerando elementos de pesos para os indicadores e para as referências de metas internas e comparativas, a alvos externos da organização. Sendo balizada 
por um conjunto de 17 indicadores que aferem a gestão e desenvolvem uma dinâmica para proposição de planos de melhorias no que tange: otimização de recursos, minimização dos pontos de geração, eficácia de ações educacionais e mudanças de procedimentos, mecanismos técnicos de tratamento e direcionamento para canais abertos de fornecedores.

Espera-se que com a referida proposta ter fomentado a literatura com um elemento decisório para balizar as medidas de gestão para as indústrias, que respondem pela maior parcela da contribuição dos resíduos perigosos brasileiros.

\section{REFERÊNCIAS}

AMARAL, S. P. Estabelecimento de indicadores e modelo de relatório de sustentabilidade ambiental, social e econômica: uma proposta para a indústria de petróleo brasileira. Tese de doutorado. Rio de Janeiro, 2003.

BORJA, P. C.; MORAES, L. R. S. Indicadores de saúde ambiental com enfoque para a área de saneamento. parte 1 - aspectos conceituais e metodológicos. Revista de engenharia sanitária e ambiental. Vol. 8 - $\mathrm{N}^{\mathrm{o}} 1$ jan/mar 2003.

BRINGHENTI, J. et al. Aplicação de indicadores no planejamento e gestão de programas de coleta seletiva de resíduos sólidos urbanos - estudo de caso. In: $23^{\circ}$ Congresso Brasileiro de Engenharia Sanitária e Ambiental. Joinvile,. Paraná. 2006.

FERREIRA, M. I. P. et al. Proposta de um sistema de indicadores ambientais e de sustentabilidade associados à cadeia produtiva de petróleo e gás. VI Congresso Nacional de excelência em gestão energia, inovação, tecnologia e complexidade para a gestão sustentável. Niterói, RJ, Brasil, agosto de 2010.

LUZ et al. Medição de desempenho ambiental baseada em método multicriterial de apoio à decisão: estudo de caso na indústria automotiva. GESTÃO \& PRODUÇÃO, v.13, n.3, p.557-570, set.-dez. 2006.

MUNCK, L. et al. Ecoeficiência: uma análise das metodologias de mensuração e seus respectivos indicadores. Revista de Gestão Social e Ambiental - RGSA, São Paulo, v. 5, n. 3, p. 183-199, set./dez. 2011.

POLAZ, C. N. M.; TEIXEIRA, B. A. N. Indicadores de sustentabilidade para a gestão municipal de resíduos sólidos urbanos: um estudo para São Carlos (SP). Revista Eng. Sanit. Ambient. v.14 n.3. jul/set 2009. 411-420.

RAMOS, M. S. Indicadores de impactos da indústria do petróleo: estudo de caso da região polarizada pelo município de Macaé. Dissertação de Mestrado, PPEA. IF Fluminense. Macaé. 2009.

RIBEIRO, D. V. A política nacional de resíduos sólidos e o uso indiscriminado de resíduos sólidos pela sociedade brasileira. Conexão Academia. A Revista Científica sobre Resíduos Sólidos Setembro 2011 - Ano I - Volume 1.

SALGADO, V. G. Proposta de Indicadores de ecoeficiencia para o transporte de gás natural. Tese de Doutorado. Rio de Janeiro, 2004.

SILVA, A. C. N. et al. Critérios adotados para seleção de indicadores de contaminação ambiental relacionados aos resíduos sólidos de serviços de saúde: uma proposta de avaliação Cad. Saúde Pública, Rio de Janeiro, 18(5):1401-1409, set-out, 2002.

SOARES, N. M. B. Gestão e gerenciamento dos resíduos sólidos domiciliares no município de Fortaleza-CE. Fortaleza: 2004. 106f. Dissertação (Mestrado) em Desenvolvimento e Meio Ambiente - Universidade Federal do Ceará.

SOUZA, J.F.; SARMENTO, R. Sistema de avaliação do desempenho ambiental de atividades petrolíferas. In: II Congresso Brasileiro de P\&D em petróleo \& gás. Rio de Janeiro. 2006. 